\title{
Successful use of posaconazole to treat invasive cutaneous fungal infection in a liver transplant patient on sirolimus
}

\author{
Randah Dahlan MBBS ${ }^{1}$, Ameen Patel MB FRCP FACP ${ }^{2}$, Shariq Haider MD FRCPC FACP 3
}

\begin{abstract}
R Dahlan, A Patel, S Haider. Successful use of posaconazole to treat invasive cutaneous fungal infection in a liver transplant patient on sirolimus. Can J Infect Dis Med Microbiol 2012;23(2):e44-e47.
\end{abstract}

Fungi are an important and common cause of cutaneous infections affecting solid organ transplant recipients. These infections can represent a primary site of infection with the potential for dissemination, or a manifestation of metastatic infection. The high morbidity and mortality associated with these infections necessitates urgent therapy with antifungal drugs; however, the interaction between these drugs and immunosuppressive therapies can be a major limitation because of drug toxicity. A case of soft tissue infection of the toe caused by Fusarium chlamydosporum and Candida guilliermondii in a liver transplant patient on sirolimus, who was successfully treated with the new antifungal agent posaconazole, is described. The pharmacokinetic interactions of sirolimus and the new triazoles, and their impact on treatment choices are briefly discussed.

\author{
L'utilisation fructueuse du posaconazole pour \\ traiter une infection fongique invasive de la \\ peau chez un greffé du foie prenant du sirolimus
}

Key Words: Cutaneous fungal infections; Liver transplantation; Posaconazole; Sirolimus

\begin{abstract}
Immunocompromised patients are at increased risk of infections from an environmental source. Fungi are increasingly recognized as major pathogens in transplant patients, with reported incidence rates of between $5 \%$ and $40 \%(1,2)$. The risk of developing fungal infections in these patients is determined by the interaction between the epidemiological exposure and the degree of immunosuppression. Fungal infections in transplant recipients are generally classified into either primary infection, with high risk for dissemination; reactivation infection, with one of the geographically restricted systemic mycosis; or opportunistic infection by fungal species that rarely cause infection in immunocompetent hosts (2). Treating these infections can be a challenge because of the difficulties encountered with the use of certain antifungal drugs and their toxicity or interaction with immunosuppressive drugs. To our knowledge, the present report is the first published case describing the use of posaconazole to treat cutaneous fungal infection in a liver transplant recipient while continuing sirolimus therapy.
\end{abstract}

\section{CASE PRESENTATION}

A 57-year-old Canadian man was referred to the infectious disease clinic in August 2007, because of painful discolouration of his right fifth toe and the lateral aspect of his left heel. These symptoms started two-and-a-half weeks before presentation and did not improve after two courses of antibiotics. He recollected walking barefoot in the soil at his cottage but did not recall any specific trauma to either foot. There were no complaints of fever, chills or night sweats. He underwent a liver transplantation in 2005 for hepatic failure secondary to BuddChiari syndrome, as well as polycythemia rubra vera and hypertension.
Post-transplant, he developed chronic kidney failure secondary to calcinuric inhibitors. His medications included sirolimus ( $2 \mathrm{mg}$ daily), mycophenolate mofetil ( $750 \mathrm{mg}$ twice daily), trimethoprim-sulfamethoxazole ( $800 \mathrm{mg} / 160 \mathrm{mg}$ every other day), hydrochlorothiazide ( $25 \mathrm{mg}$ daily) and warfarin (9 mg daily). He had no known drug allergies and his family and social history were unremarkable.

On examination, he appeared systemically well, was afebrile and had a heart rate of 78 beats/min, a respiratory rate of 18 breaths/min and an oxygen saturation of $98 \%$ on room air. His right fifth toe was purple, mottled and ulcerated with involvement of the nail plate (Figure 1). There was no discharge, but the toe was exquisitely tender to palpation. Mild bilateral ankle edema was present.

The investigations included normal electrolytes, and a white blood cell count of $9.8 \times 10^{9} / \mathrm{L}$ with a lymphocyte count of $1.4 \times 10^{9} / \mathrm{L}$. His urea and creatinine levels were $8.0 \mathrm{mmol} / \mathrm{L}$ and $139 \mathrm{mmol} / \mathrm{L}$, respectively. He had a normal foot and ankle $\mathrm{x}$-ray, and a magnetic resonance imaging scan of his feet showed no evidence of osteomyelitis. A skin biopsy of the right fifth toe showed parakeratosis, mild acanthotic hyperplasia with reactive atypia, microvascular dilation and mild lymphocytic infiltration of the upper dermis, changes consistent with a dermatitis reaction with no evidence of neoplasm. However, mycology culture and susceptibility testing identified Candida guilliermondii and Fusarium chlamydosporum, which are recognized pathogens in the context of cutaneous infections with nail involvement in transplant recipients, as seen in our patient, rather than a simple colonizer. The susceptibility results were obtained using the YeastOne (TREK Diagnostic Systems, Inc, USA) method for C guilliermondii, and the

${ }^{1}$ Department of Internal Medicine, McMaster University; ${ }^{2}$ Division of General Internal Medicine; ${ }^{3}$ Division of Infectious Diseases, Hamilton Health Sciences, Hamilton, Ontario

Correspondence: Dr Randah Dahlan, Department of Internal Medicine, McMaster University, 1200 Main Street West, Hamilton, Ontario

L8S 4L8. Telephone 905-521-2100, e-mail randaad@hotmail.com 

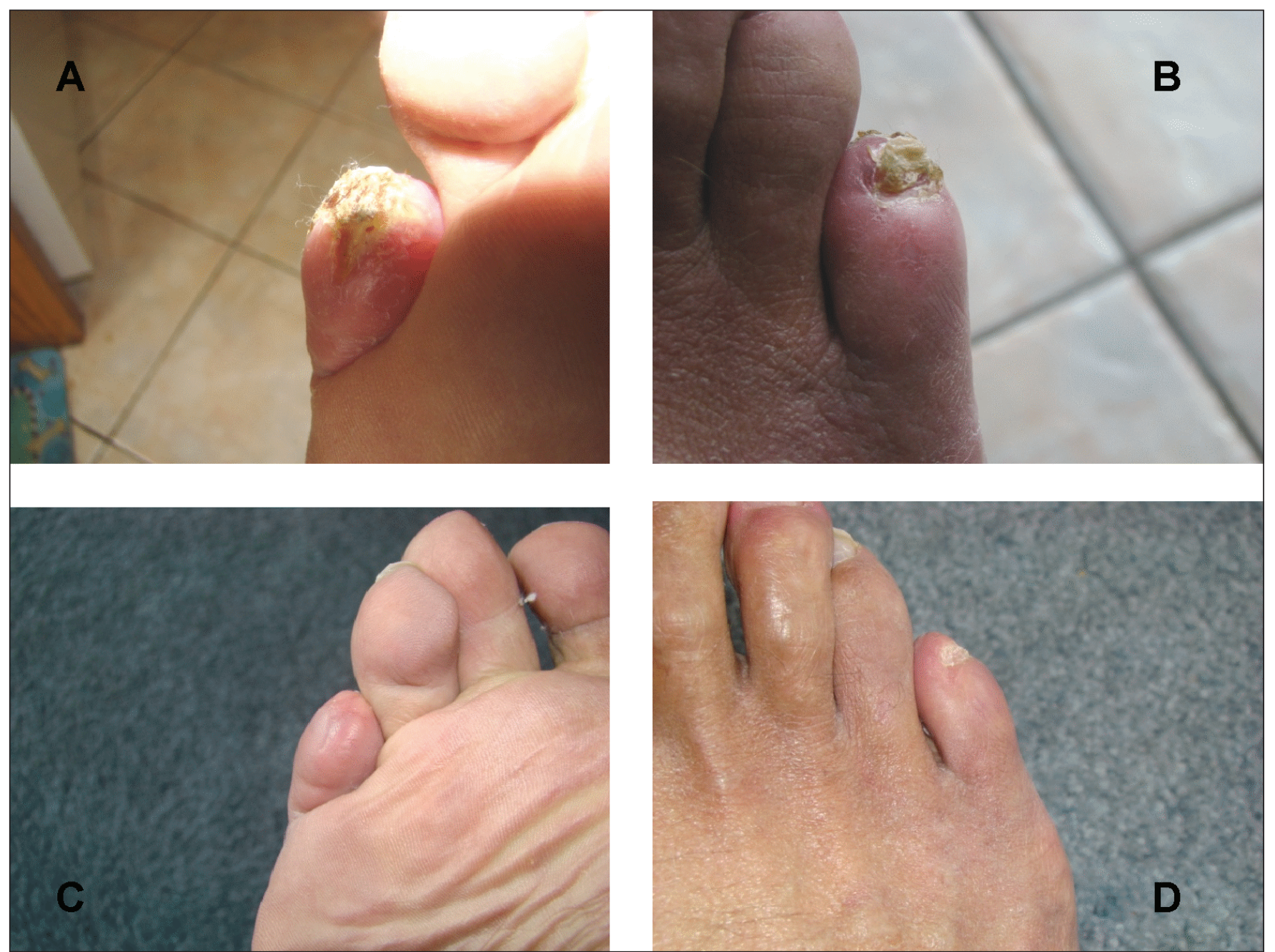

Figure 1) A and B Right fifth toe at time of presentation with swelling, discolouration and ulceration. $\mathbf{C}$ and $\mathbf{D}$ Right fifth toe after treatment with complete healing

TABLE 1

Results of the mycology culture and the susceptibility profile from the biopsy of the right fifth toe

\begin{tabular}{|c|c|c|c|}
\hline \multicolumn{2}{|c|}{ Fusarium chlamydosporum } & \multicolumn{2}{|c|}{ Candida guilliermondii } \\
\hline Antifungal drug & MIC, mg/L & Antifungal drug & MIC, mg/L \\
\hline Amphotericin B & 0.5 & Amphotericin B & 0.5 \\
\hline Fluconazole & 32.0 & Fluconazole & 2 \\
\hline Ketoconazole & 1.0 & 5-fucytosine & 0.03 \\
\hline Itraconazole & 2.0 & Itraconazole & 0.25 \\
\hline \multirow[t]{2}{*}{ Voriconazole } & 0.5 & Voriconazole & 0.12 \\
\hline & & Gaspofungin & 0.25 \\
\hline
\end{tabular}

MIC Minimum inhibitory concentration

Clinical_and Laboratory Standards Institute method for F chlamydosporum (Table 1). Susceptibility to posaconazole was not performed because it was not a routine test at that time.

The lipid formulation of amphotericin B could not be used due to concern of worsening of the patient's chronic renal insufficiency, and voriconazole was relatively contraindicated at that time because the patient was on sirolimus. As an alternative, the new antifungal agent posaconazole (400 mg orally twice daily) was used, and the patient was instructed to take it with meals. The sirolimus dose was reduced to $1 \mathrm{mg}$ daily then to $1 \mathrm{mg}$ every other day, and drug levels were followed closely by the patient's transplant centre to compensate for the cytochrome P450 (CYP) interaction between sirolimus and posaconazole. During the course of therapy, liver function tests remained normal and the soirlimus levels were stable between $3.6 \mu \mathrm{g} / \mathrm{L}$ and $5.3 \mu \mathrm{g} / \mathrm{L}$. Posaconazole drug level was not tested. The patient received a sixmonth course of posaconazole with complete recovery (Figure 1).

\section{DISCUSSION}

Fungal infections are occurring with increasing frequency secondary to medical advances in the areas of transplantation, cancer management and autoimmune diseases. Yeasts most frequently isolated in clinical practice are Candida species and Cryptococcus species (3). $F$ chlamydosporum and $\mathrm{C}$ guilliermondii are uncommon pathogens that can present with cutaneous infections with a potential risk for dissemination in immunocompromised hosts. C guilliermondii has been isolated from numerous human cutaneous infections but systemic infections are rare. Fusarium species are soil saprophytes that use the skin as a portal of entry causing soft tissue infection including superficial (onychomycosis and keratitis), locally invasive or disseminated infections (4). Inhalation of spores is another major portal of entry, leading to fungal sinusitis and pneumonia. A previous review of 259 patients with fusarial infection showed that $72 \%$ of immunocompromised hosts had skin involvement, which was described as papules, nodules with or without necrosis and gangrenous changes, cellulitis, onychomycosis, ulcers, keratotic lesions or subcutaneous abscesses (5). The presentation varies according to the extent of infection (localized versus disseminated) and the presence or absence of a history of skin breakdown. Skin involvement was associated with a higher mortality rate (70\%), particularly with patients whose lesions were disseminated (5). 
TABLE 2

Comparative pharmacokinetics of different triazoles and sirolimus

\begin{tabular}{|c|c|c|c|c|c|}
\hline Agent & Dosage form & Metabolism (\%) & Bioavailability, \% & Cytochrome P4503A4 & Elimination (\%) \\
\hline Fluconazole & Intravenous, oral & Hepatic (20) & 95 & Substrate & Renal (80) \\
\hline Itraconazole & Intravenous, oral & Hepatic (extensive) & 50 & Substrate and inhibitor & Hepatic \\
\hline Posaconazole & Oral & Hepatic $(<30)$ & Variable & Inhibitor & Feces (77) \\
\hline Voriconazole & Intravenous, oral & Hepatic (98) & 98 & Substrate and inhibitor & Renal (83) \\
\hline Sirolimus & Oral & Hepatic (extensive) & 15 & Substrate & Feces (91) \\
\hline
\end{tabular}

Early identification of localized skin disease and institution of therapy with debridement, antifungal agents, or both are crucial and can be life-saving. Despite the reported efficacy of liposomal amphotericin B in treating fusarial skin infection in a liver transplant patient (6), it could not be used in our case because of impaired renal function, and the only other option was to use triazole agents. No two triazole agents offer the same pharmacodynamic profile and choosing among different agents can be difficult (7) (Table 2). Newer azole agents (voriconazole and posaconazole) are emerging as first-line therapies for severe fungal infections because of superior pharmacokinetics and pharmacodynamics, improved safety profiles and clinical efficacy in the treatment of systemic and localized mycoses (7-9).

Posaconazole, as with other triazole antifungal agents, works by binding to the heme cofactor located on the active site of the cytochrome CYP-dependent 14-alpha-demethylase. This enzyme is responsible for the conversion of lanosterol to 14-alpha-dimethyl lanosterol in the ergosterol biosynthetic pathway. Ergosterol is an essential component of the fungal cell membrane. The net result is disruption of the integrity and function of the fungal cell membrane, as well as inhibition of fungal growth. Posaconazole is available only in oral formulation (trials of intravenous formulations are upcoming), and its absorption is highly enhanced by concurrent food intake. Therefore, it should be administered during or within 20 min following a full meal or an oral liquid nutritional supplement. Posaconazole is primarily eliminated via the fecal route, and approximately $14 \%$ of an administered dose undergoes non-CYP hepatic metabolism to inactive metabolites and is excreted in urine (10). Therefore, only minimal amounts are recovered in the urine and dose reductions are not required for patients with chronic kidney disease. Gastrointestinal symptoms, including diarrhea, nausea and abdominal pain are the most common side effects of this drug and occur in approximately $1 \%$ to $10 \%$ of users (10).

Although posaconazole does not undergo any appreciable phase I biotransformation reactions, it is an inhibitor of CYP3A4 metabolism and is a substrate and inhibitor of $\mathrm{P}$ glycoprotein transport (Table 2). Drug-drug interactions similar to those seen with other inhibitors of CYP3A4 can occur $(11,12)$. For example, sirolimus is metabolized by cytochrome CYP3A4 and is a substrate of the P glycoprotein drug efflux pump. Patients on sirolimus may have up to a sevenfold increase in trough level when treated with drugs such as triazoles because of a decrease in the metabolic activity of this enzymatic system (13). Moton et al (14) evaluated the effects of posaconazole on sirolimus pharmacokinetics in 12 healthy subjects and found that coadministration of these two drugs can lead to an increase in sirolimus Cmax and area under the curve (AUC) by 6.7- and 8.9-fold, respectively. Therefore, it is essential to monitor blood sirolimus concentrations and to adjust the sirolimus doses before and after coadministration of these drugs. A recent in vitro study assessing drug interactions found significant antagonism with the concurrent use of sirolimus and posaconazole (15).

In comparison, voriconazole is both a substrate and an inhibitor of CYP3A4 with a net effect of increasing sirolimus's AUC. This effect is not easily managed by dose adjustment due to its unpredictable nonlinear pharmacokinetics. In addition, voriconazole is also metabolized by other isoenzymes, namely CYP2C19 and CYP2C9. The latter can exhibit some genetic polymorphisms leading to subject-to-subject variability in voriconazole pharmacokinetics. However, the safety of coadministration of sirolimus and voriconazole has been previously reported with an initial empirical 90\% sirolimus dose reduction combined with systematic monitoring of trough levels (16). In addition, a pilot study (17) assessing the concurrent use of sirolimus and voriconazole confirmed the safety of coadministration, provided that the sirolimus drug level is closely monitored.

\section{CONCLUSION}

The new triazole antifungal drugs offer an expanded spectrum of activity over other members of its class, and understanding subtle differences among drugs within this class is essential to safely and effectively using these agents. We described a case of invasive cutaneous fungal infection in a liver transplant recipient on sirolimus that was successfully treated with posaconazole.

\section{REFERENCES}

1. Paya CV. Fungal infections in solid-organ transplantation. Clin Infect Dis 1993;16:677-88.

2. Hong W, Wen H, Liao W. Fungal infection in organ transplant patients. Chin Med J (Eng) 2003;116:1421-5.

3. Enoch DA, Ludlam HA, Brown NM. Invasive fungal infections: a review of epidemiology and management options. J Med Microbiol 2006;55:809-18.

4. Nucci M, Anaissie E. Fusarium infections in immunocompromised patients. Clin Microbiol Rev 2007;20:695-704.

5. Nucci M, Anaissie E. Cutaneous infection by Fusarium species in healthy and immunocompromised hosts: implications for diagnosis and management. Clin Infects Dis 2002;35:909-20.

6. Halpern M, Balbi E, Carius L, et al. Cellulitis and nodular skin lesions due to Fusarium spp. in liver transplant: Case report. Transplant Proc 2010;42:599-600.

7. Saad A, DePestel D, Carver P. Factors influencing the magnitude and clinical significance of drug interactions between azole antifungals and select immunosuppressants. Pharmacotherapy 2006;26:1730-44.

8. Pfaller MA, Messer SA, Hollis RJ, Jones RN; SENTRY Participants Group. Antifungal activities of posaconazole, ravuconazole, and voriconazole compared to those of itraconazole and amphotericin B against 239 clinical isolates of Aspergillus spp. and other filamentous fungi: Report from SENTRY Antimicrobial Surveillance Program, 2000. Antimicrob Agents Chemother 2002;46:1032-37.

9. Pasqualotto AC, Denning DW. New and emerging treatments for fungal infections. J Antimicrob Chemother 2008;61(Suppl 1)1:119-30.

10. Krieter P, Flannery B, Musick T, Gohdes M, Martinho M, Courtney R. Disposition of posaconazole following single-dose oral administration in healthy subjects. Antimicrob Agents Chemother 2004;48:3543-51.

11. Schiller DS, Fung HB. Posaconazole: An extended-spectrum triazole antifungal agent. Clin Ther 2007;29:1862-86.

12. Sansone-Parsons A, Krishna G, Martinho M, Kantesaria B, Gelone S, Mant TG. Effect of oral posaconazole on the pharmacokinetics of cyclosporine and tacrolimus. Pharmacotherapy 2007;27:825-34.

13. Sadaba B, Campanero MA, Quetglas EG, Azanza JR. Clinical relevance of sirolimus drug interactions in transplant patients. Transplant Proc 2004;36:3226-8.

14. Moton A, Ma L, Krishna G, Martinho M, Seiberling S, McLeod J. Effects of oral posaconazole on the pharmacokinetics of sirolimus. Curr Med Res Opin 2009;25:701-7. 
15. Narreddy S, Manavathu E, Chandrasekar P, Alangaden G, Revankar S. In vitro interaction of posaconazole with calcineurin inhibitors and sirolimus against zygomycetes. J Antimicrob Chemother 2010;65:701-3.

16. Marty FM, Lowry CM, Cutler CS, et al. Voriconazole and sirolimus co-administration after allogeneic hematopoietic stem cell transplantation. Biol Blood Marrow Transplant 2006;12:552-9.
17. Surowiec D, DePestel D, Carver P. Concurrent administration of sirolimus and voriconazole: A pilot study assessing safety and approaches to appropriate management. Pharmacotherapy 2008;28:719-29. 


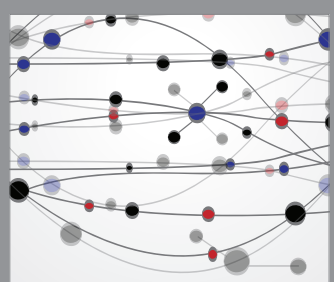

The Scientific World Journal
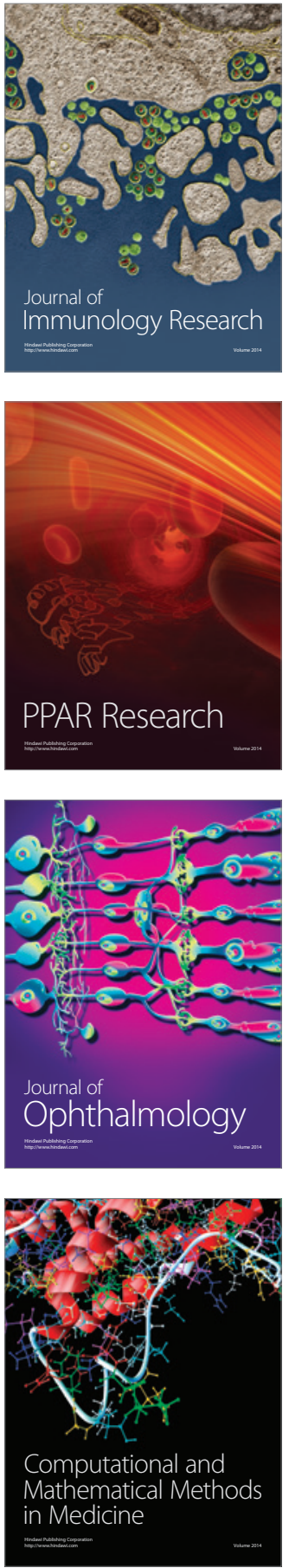

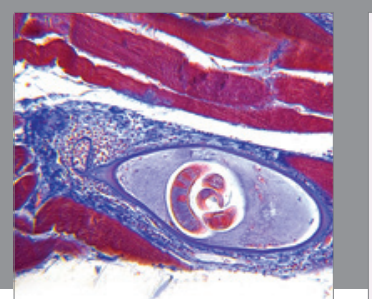

Gastroenterology Research and Practice

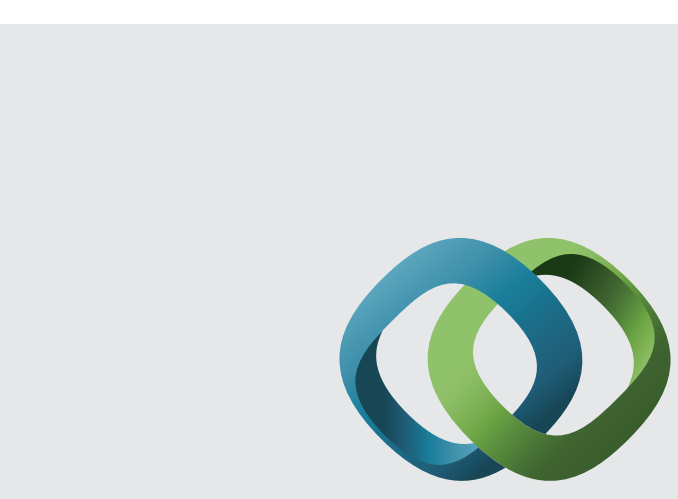

\section{Hindawi}

Submit your manuscripts at

http://www.hindawi.com
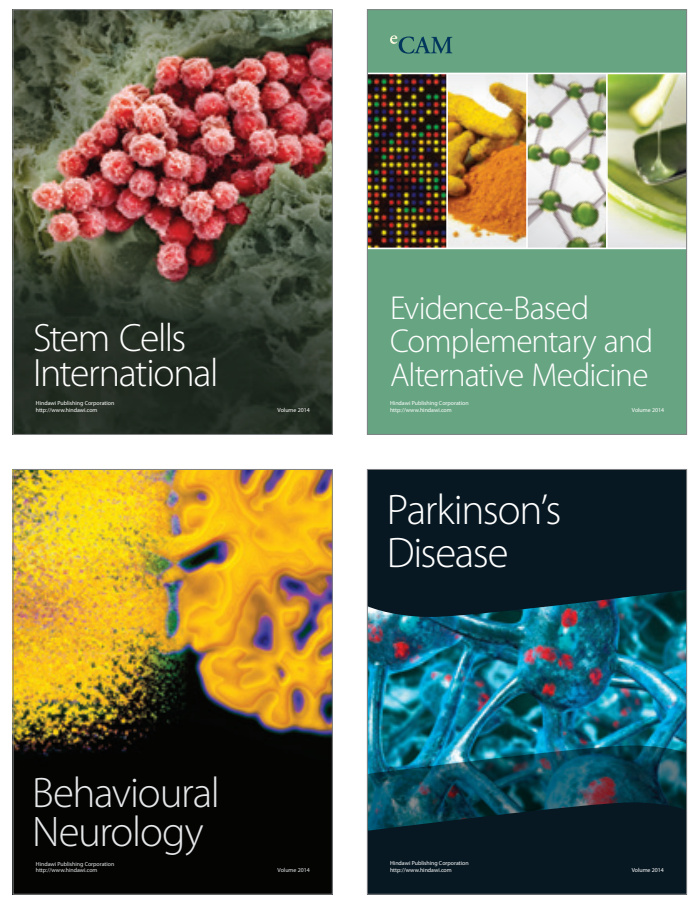
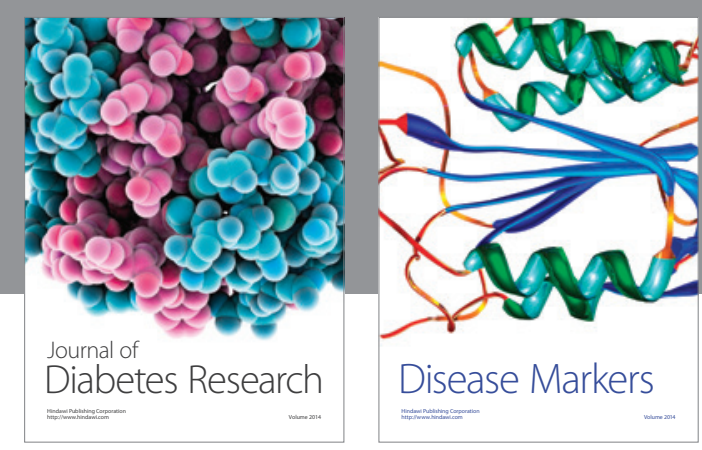

Disease Markers
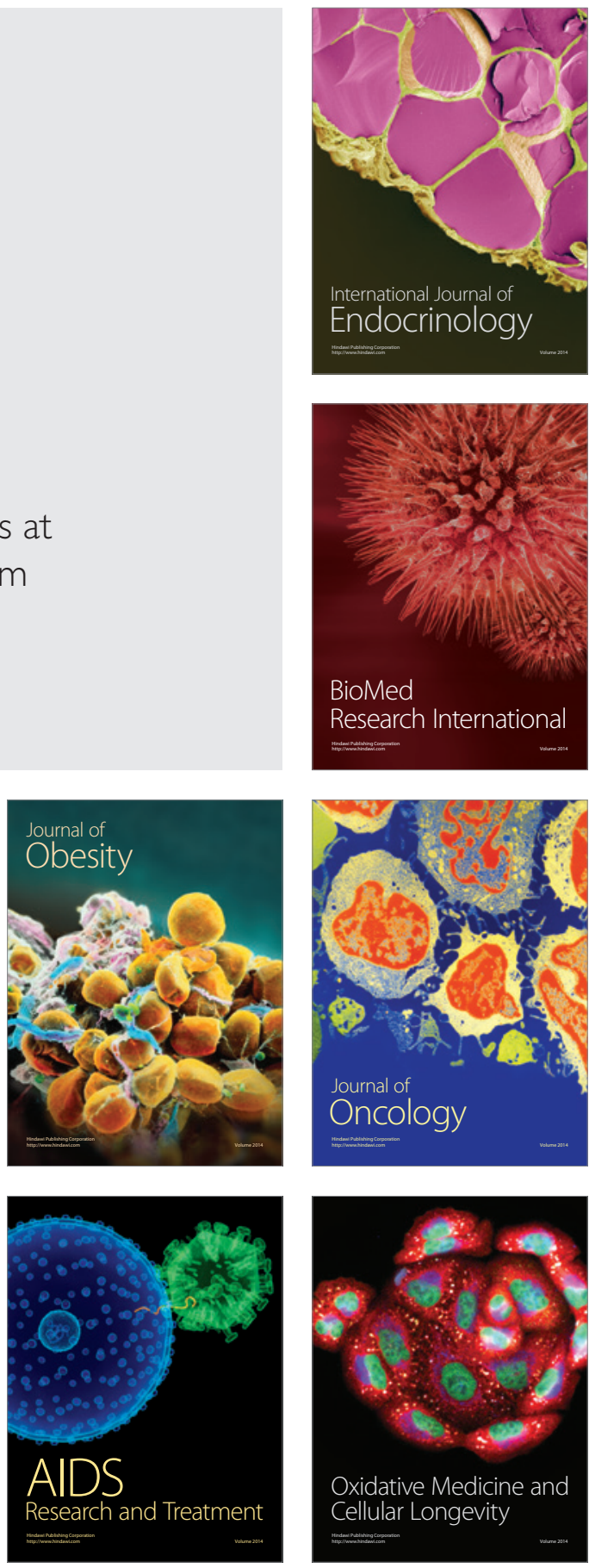